\title{
Can extreme black holes have (long) Abelian Higgs hair?
}

\author{
A. Chamblin, ${ }^{1,4}$ J. M. A. Ashbourn-Chamblin, ${ }^{2}$ R. Emparan, ${ }^{3}$ and A. Sornborger ${ }^{4}$ \\ ${ }^{1}$ Institute for Theoretical Physics, University of California, Santa Barbara, California 93106-4030 \\ ${ }^{2}$ Wolfson College, University of Oxford, Oxford OX2 6UD, England \\ ${ }^{3}$ Department of Physics, University of California, Santa Barbara, California 93106 \\ ${ }^{4}$ DAMTP, Silver Street, Cambridge, CB3 9EW, England \\ (Received 12 May 1998; published 17 November 1998)
}

\begin{abstract}
It has been argued that a black hole horizon can support the long-range fields of a Nielsen-Olesen string and that one can think of such a vortex as black hole "hair.' In this paper, we examine the properties of an Abelian Higgs vortex in the presence of a charged black hole as we allow the hole to approach extremality. Using both analytical and numerical techniques, we show that the magnetic field lines (as well as the scalar field) of the vortex are completely expelled from the black hole in the extreme limit. This was to be expected, since extreme black holes in Einstein-Maxwell theory are known to exhibit such a 'Meissner effect', in general. This would seem to imply that a vortex does not want to be attached to an extreme black hole. We calculate the total energy of the vortex fields in the presence of an extreme black hole. When the hole is small relative to the size of the vortex, it is energetically favored for the hole to remain inside the vortex region, contrary to the intuition that the hole should be expelled. However, as we allow the extreme horizon radius to become very large compared to the radius of the vortex, we do find evidence of an instability. This proves that it is energetically unfavorable for a thin vortex to interact with a large extreme black hole. This would seem to dispel the notion that a black hole can support "long" Abelian Higgs hair in the extreme limit. We show that these considerations do not go through in the near-extreme limit. Finally, we discuss the implications for strings that end at black holes, as in the processes where a string snaps by nucleating black holes. [S0556-2821(98)08320-9]
\end{abstract}

PACS number(s): 04.40.-b, 04.70.-s, 11.27.+d, 98.80.Cq

\section{INTRODUCTION}

Black hole "hair" is defined to be any field(s) associated with a stationary black hole configuration which can be detected by asymptotic observers, but which cannot be identified with the electromagnetic or gravitational degrees of freedom. Back in the heyday of black hole physics, a number of results were proved $[1,2,3]$ which seemed to imply that black holes "have no hair." Put more colloquially, these results implied that given certain assumptions the only information about a black hole which an observer far from the hole can determine experimentally is summarized by the electric charge, magnetic charge, angular momentum, and mass of the hole. Such uniqueness results are referred to as "nohair" theorems. These celebrated results would seem to imply that a black hole horizon can support only these limited gauge charges; for a long time, physicists thought that other matter fields simply could not be associated with a black hole. However, this prejudice was to some extent discredited when Bartnik and McKinnon [4] discovered a solution of the Einstein-Yang-Mills equations which had "particle"-like quantum numbers which did not correspond to the gravitational or Maxwell fields. More precisely, the holes of [4] support Yang-Mills fields which can be detected by asymptotic observers; one therefore says that these black holes are colored.

Of course, these exotic solutions do not impugn the original no-hair results since all such solutions are known to be linearly unstable (see, e.g., [5]). These colored holes are therefore said to "evade" the usual no-hair results.

There are other amusing tricks which allow one to evade no-hair theorems. For example, the reader will recall [6] that in string theory the Einstein equations are induced from the low-energy effective field theory only to "zeroth" order in $\alpha^{\prime}$, where $\alpha^{\prime}$ denotes the Regge slope of string theory. If you include the order- $\alpha^{\prime}$ corrections, then you get curvature"squared" terms in the Lagrangian (you also get the usual dilatonic terms). It turns out [7] that black hole solutions in such a curvature-squared, higher-derivative theory of gravity can support nontrivial dilatonic configurations outside the horizon, and so they are said to possess "dilatonic" hair. Again, these results do not actually contradict the original no-hair theorems since they only apply in exotic situations.

What these results teach us is that we have to tread very carefully whenever we start talking about black hole hair. We will stick with our definition of hair as any property which can be measured by asymptotic observers. Furthermore, we shall follow [8] and use the term "dressing" for the question of whether or not fields actually reside on the horizon.

With all of this in mind, we now want to analyze the extent to which hair is present in situations where we allow the topology of some field configurations to be nontrivial; in particular, an interesting question is whether or not topological defects, such as domain walls, strings, or textures [9], can act as "hair" for a black hole. In [8] evidence was presented that a Nielsen-Olesen [U(1)] vortex can act as "long" hair for a Schwarzschild black hole. More precisely, in [8] the authors studied the problem of whether or not such a vortex can exist on a Schwarzschild black hole background (neglecting at first the gravitational back reaction); they presented analytical and numerical evidence for such a solution. They went on to include the gravitational back reaction of a single thin vortex and managed to rederive the "Aryal-Ford- 
Vilenkin" (AFV) metric [10], which is a solution meant to model a cosmic string threading through a Schwarzschild black hole (i.e., the AFV solution is just a conical defect centered on a black hole). Thus they were able to argue that the AFV solution truly is the "thin vortex" limit of a "physical" vortex-black-hole configuration. Using all of these results, they concluded with an argument that the Abelian Higgs vortex is not just dressing for the Schwarzschild black hole, but rather that the vortex is truly hair, that is, a property of the black hole which can be detected by asymptotic observers.

In this paper, we extend the analysis of [8] and allow the black hole to be charged. That is to say, we consider the problem of an Abelian Higgs vortex in the ReissnerNordström background. In order to "turn up" the electric charge of the hole, we have to allow for the presence of two $\mathrm{U}(1)$ 's [one U(1) is where the charge of the hole resides and the other $\mathrm{U}(1)$ is the symmetry spontaneously broken in the ground state]; otherwise, the charge would be screened. We find that the results of [8] are reproduced when the charge of the hole is very small relative to the mass. However, as we increase the charge and the hole approaches extremality, we find that something very remarkable happens. In the extreme limit, all of the fields associated with the vortex (both the magnetic and scalar degrees of freedom) are expelled from the horizon of the black hole. We present dramatic numerical evidence that the magnetic and scalar fields always "wrap around" the horizon in the extremal limit. This behavior was expected, given that extreme black holes in EinsteinMaxwell-dilaton theories generically display such a "Meissner effect" and so can be thought of as "superconductors" (a deeper analysis of the superconducting properties of extremal black holes and $p$-branes in Kaluza-Klein and string theories will be given in [11]).

We go on to calculate the total energy present in the electromagnetic field (of the vortex) as we allow the extreme black hole to become very large compared to the size of the vortex, and we find an instability. Put more simply, for black holes large compared to the vortex radius, the energy of a vortex which does not wrap the hole (i.e., with the black hole outside the vortex) is much less than the energy of a vortex which does wrap the hole. It is therefore energetically unfavorable for the vortex to interact with the hole, and indeed the vortex will want to "slide" off of the hole. Thus, in the thin vortex limit, a vortex does not want to be attached to an extreme black hole. It follows that the vortex cannot in any way be thought of as a "property of the black hole which can be measured at infinity"; in other words, an Abelian Higgs vortex is not hair for an extreme black hole. Curiously, the expulsion of the vortex does not proceed gradually as the black hole approaches extremality; rather, we have found numerical evidence that a nonextreme black hole is always pierced by a vortex, no matter how close to extremality it is. We conclude with a discussion of the implications of our results to scenarios involving strings ending on black holes, in particular, the snapping of strings by the formation of black hole pairs.

\section{NIELSEN-OLESEN VORTEX IN THE PRESENCE OF A CHARGED BLACK HOLE}

In this section we analyze the Nielsen-Olesen equations for an Abelian Higgs vortex [12] in the Reissner-Nordström background. Since we want to provide some continuity with the study of Achúcarro et al. [8], which in some respects we generalize, we will present our analysis in a form and notation that closely parallel theirs.

Our treatment of the black-hole-string-vortex system involves a clear separation between the degrees of freedom of each of these objects. The action takes the form

$$
S=S_{1}+S_{2},
$$

where the first term is an Einstein-Hilbert-Maxwell action,

$$
S_{1}=\frac{1}{16 \pi G} \int d^{4} x \sqrt{-g}\left(-R-\frac{1}{4} \mathcal{F}^{2}\right),
$$

and the second describes an Abelian Higgs system minimally coupled to gravity:

$S_{2}=\int d^{4} x \sqrt{-g}\left(D_{\mu} \Phi^{\dagger} D^{\mu} \Phi-\frac{1}{4 e^{2}} F^{2}-\frac{\lambda}{4}\left(\Phi^{\dagger} \Phi-\eta^{2}\right)^{2}\right)$.

The matter content of the Abelian Higgs system consists of the complex Higgs field $\Phi$ and a U(1) gauge field with strength $F_{\mu \nu}$ and potential $A_{\mu}$. Both the Higgs scalar and the gauge field become massive in the broken symmetry phase. They are coupled through the gauge covariant derivative $D_{\mu}=\nabla_{\mu}+i A_{\mu}$, where $\nabla_{\mu}$ is the spacetime covariant derivative. As in [8], we choose metric signature $(+---)$.

The degrees of freedom in $S_{2}$ will be treated as "test fields"; i.e., their energy-momentum tensor is supposed to yield a negligible contribution to the source of the gravitational field. The latter, instead, affects the propagation of the fields $\Phi$ and $A_{\mu}$ : an exact solution of the EinsteinMaxwell equations from $S_{1}$ will be plugged into the Abelian Higgs action $S_{2}$ as a fixed, background metric $g_{\mu \nu}$. Notice that we have two different gauge fields, $\mathcal{F}$ and $F$, and each is treated in a very different manner. It is only $F$ that couples to the Higgs field and is therefore subject to spontaneous symmetry breaking. The other gauge field $\mathcal{F}$ could be thought of as the free, massless Maxwell field of everyday experience; apart from modifying the background geometry, its dynamics will be of little concern to us here. Notice that whereas we treat $F$ as a test field, the back reaction of $\mathcal{F}$ on the geometry will be fully accounted for.

The parameter $\eta$ is the energy scale of symmetry breaking and $\lambda$ is the Higgs coupling. These can be related to the Higgs boson mass by $m_{\text {Higgs }}=\eta \sqrt{\lambda}$. There is another relevant mass scale, i.e., that of the vector field in the broken phase, $m_{\text {vector }}=\sqrt{2} e \eta$. On length scales smaller than $m_{\text {vector }}^{-1}, m_{\text {Higgs }}^{-1}$, the vector and Higgs fields behave as essentially massless. It is also convenient to define the Bogomolnyi parameter $\beta=\lambda / 2 e^{2}=m_{\text {Higgs }}^{2} / m_{\text {vector }}^{2}$.

The action (2.3) has a $\mathrm{U}(1)$ invariance realized by 


$$
\Phi \rightarrow \Phi e^{i \Lambda(x)}, \quad A_{\mu} \rightarrow A_{\mu}-\nabla_{\mu} \Lambda(x)
$$

which is spontaneously broken in the ground state, $\Phi$ $=\eta e^{i \Lambda_{0}}$. Besides this ground state, another solution, the vortex, is present when the phase of $\Phi(x)$ is a nonsingle-valued quantity. To better describe this, define the real fields $X, P_{\mu}$, and $\chi$ by

$$
\Phi=\eta X e^{i \chi}, \quad A_{\mu}=P_{\mu}-\nabla_{\mu} \chi .
$$

A vortex is present when $\oint d \chi=2 \pi N$, the integer $N$ being called the winding number of the vortex. If $N \neq 0$ and if the spatial topology is trivial, then, by continuity, the integration loop must encircle a point of unbroken symmetry $(X=0)$, namely, the vortex core.

The Euler-Lagrange equations that follow by varying $X$ in the action (2.3) are

$$
\nabla^{2} X-X P_{\mu} P^{\mu}+\frac{\lambda \eta^{2}}{2} X\left(X^{2}-1\right)=0
$$

while by varying $A_{\mu}$ one finds

$$
\nabla_{\mu} F^{\mu \nu}+2 e^{2} \eta^{2} X^{2} P^{\nu}=0 .
$$

The field $\chi$ is not dynamical. In flat space, vortices of Nielsen-Olesen type [12] appear as cylindrically symmetric solutions

$$
\Phi=X\left(r_{c}\right) e^{i N \varphi}, \quad P_{\varphi}=N P\left(r_{c}\right),
$$

$r_{c}$ being the cylinder radial coordinate and all other components of $P_{\mu}$ being zero. We will be concerned, however, not with flat space, but with another solution of the EinsteinMaxwell theory (2.2), namely, the Reissner-Nordström black hole

$$
\begin{aligned}
d s^{2} & =V d t^{2}-\frac{d \rho^{2}}{V}-\rho^{2}\left(d \theta^{2}+\sin ^{2} \theta d \varphi^{2}\right), \\
V & =1-\frac{2 G m}{\rho}+\frac{q^{2}}{\rho^{2}}
\end{aligned}
$$

(the charge $q$ is measured here in geometrical units), which is not cylindrically symmetric. This makes the analysis of the solutions somewhat more complicated.

It will be convenient to rescale the radial coordinate and black hole parameters by the Higgs wavelength to work with the nondimensional variables $(r, E, Q)=\eta \sqrt{\lambda}(\rho, G m, q)$. In terms of these variables,

$$
V=1-\frac{2 E}{r}+\frac{Q^{2}}{r^{2}} .
$$

We stress that the charge $Q$ of the black hole, which couples to the field $\mathcal{F}$, is unrelated to the Abelian gauge field $F$ associated with the vortex. $Q$ can be primarily thought of as a parameter that allows us to modify the background geometry, in particular, to consider the extremal black hole backgrounds described below.
The Reissner-Nordström black hole has inner and outer horizons where $V(r)=0$. We will only be interested in the outer horizon, which is at radius

$$
r_{+}=E+\sqrt{E^{2}-Q^{2}} .
$$

The horizon exists as long as $E \geqslant|Q|$; otherwise, one finds a naked singularity. If the inequality is saturated, $r_{+}=E$ $=|Q|$, then $V(r)$ has a double zero at $r_{+}$and the black hole is said to be extremal.

Return now to the equations of the vortex. One can consistently take

$$
X=X(r, \theta), \quad P_{\varphi}=N P(r, \theta),
$$

which simplifies the equations of motion (2.6), (2.7), to the form

$$
\begin{gathered}
-\frac{1}{r^{2}} \partial_{r}\left(r^{2} V \partial_{r} X\right)-\frac{1}{r^{2} \sin \theta} \partial_{\theta}\left(\sin \theta \partial_{\theta} X\right) \\
+\frac{1}{2} X\left(X^{2}-1\right)+\frac{N^{2} X P^{2}}{r^{2} \sin ^{2} \theta}=0 \\
\partial_{r}\left(V \partial_{r} P\right)+\frac{\sin \theta}{r^{2}} \partial_{\theta}\left(\frac{\partial_{\theta} P}{\sin \theta}\right)-\frac{X^{2} P}{\beta}=0 .
\end{gathered}
$$

In this generic form these equations allow us to recover two interesting situations as limiting cases. First, when $\beta \rightarrow \infty$ the Higgs field decouples. In this situation we would be essentially studying a free Maxwell test field in the ReissnerNordstrom background. The complementary situation arises when $P=1$ (a constant) throughout the space: this would be a global string, i.e., without any local gauge dynamics, in the presence of the charged hole.

Equations (2.13), (2.14), are, in general, rather intractable in exact form and we will need to resort to approximation methods. In the next section, we will solve the equations numerically and study configurations with arbitrary relative sizes of the black-hole-vortex radii. For the remainder of this section we will describe an analytical solution of these equations for the case where the black hole is small relative to the vortex size. In the units we are using the radius of the flux tube is $r \sim \sqrt{2 N} \beta^{1 / 4}$ for $N \gg 1$. Thus we will require $\sqrt{N} \gg E$. This sort of large- $N$ limit was first employed to obtain analytical results in [13]. The results we obtain in this way will be consistent with our numerical solutions in the next section.

Well inside the core of the vortex the gauge symmetry remains essentially unbroken. Thus we expect $X \approx 0$ or, better, $X^{2} / \beta \approx 0$. It is not difficult to see that, within the approximation considered, one can consistently neglect the last term in Eq. (2.14) and then attempt to solve

$$
\partial_{r}\left(V \partial_{r} P\right)+\frac{\sin \theta}{r^{2}} \partial_{\theta}\left(\frac{\partial_{\theta} P}{\sin \theta}\right) \approx 0 .
$$


For the Schwarzschild background $(Q=0)$, a solution is provided by $P-1 \propto r^{2} \sin ^{2} \theta$. This suggests that we try the ansatz

$$
P \approx 1+a(r) \sin ^{2} \theta
$$

The equation we must solve now is

$$
\left(r^{2}-2 E r+Q^{2}\right) a^{\prime \prime}+2\left(E-\frac{Q^{2}}{r}\right) a^{\prime}-2 a=0,
$$

which admits the solution

$$
a(r)=-p\left(r^{2}-Q^{2}\right),
$$

and hence

$$
P \approx 1-p\left(r^{2}-Q^{2}\right) \sin ^{2} \theta
$$

Here $p$ is an integration constant equal to twice the magnetic field strength at the center of the core. We have also chosen the parameters in order to have $P \rightarrow 1$ at the string axis ( $\theta$ $=0, \pi)$. Far from the black hole, but still inside the vortex, we can perform an analysis similar to that in [13] to show that

$$
p \approx \frac{1}{2 N \sqrt{\beta}}
$$

Large $N$ thus means small $p$.

Now we have to solve the equation for the Higgs field $X$, Eq. (2.13). Following [13], we set $X=\xi^{N}$ and expand in powers of $1 / N$. This yields

$$
V\left(\frac{\partial_{r} \xi}{\xi}\right)^{2}+\frac{1}{r^{2}}\left(\frac{\partial_{\theta} \xi}{\xi}\right)^{2}=\frac{P^{2}}{r^{2} \sin ^{2} \theta}+O\left(1 / N^{2}\right) .
$$

To be consistent we must neglect the terms proportional to $p^{2}$, since as we have seen they would contribute to $O\left(1 / N^{2}\right) .{ }^{1}$ Having done this, the equation becomes separable and can be solved in the form $\xi=b(r) \sin \theta$, where $b$ must satisfy

$$
\frac{b^{\prime}}{b}=\frac{1-p\left(r^{2}-Q^{2}\right)}{r \sqrt{V}} .
$$

This is integrated to yield

$$
\begin{aligned}
b(r)= & k(r-E+r \sqrt{V}) \\
& \times \exp \left(-\frac{p}{2}\left(r^{2}+3 E r\right) \sqrt{V}-\frac{3}{2} p\left(E^{2}-Q^{2}\right)\right. \\
& \times \ln (r-E+r \sqrt{V}))
\end{aligned}
$$

\footnotetext{
${ }^{1}$ This limits the validity of the solution to distances $r \sin \theta$ sufficiently smaller than $\sqrt{2 N}$.
}

( $k$ is another integration constant; its precise value is irrelevant for our purposes). From here we get $X$ as

$$
X \approx b^{N}(r) \sin ^{N} \theta .
$$

Equations (2.19), (2.23), and (2.24), constitute our solution describing a "test vortex" residing in the background of a charged black hole that sits well within the vortex core. The presence of charge induces a number of qualitative changes in the picture described in [8] (the neutral case). To start with, notice that the distance at which $P \approx 0$, which roughly defines the thickness of the vortex, is

$$
r \sin \theta \approx \sqrt{\frac{1}{p}+Q^{2} \sin ^{2} \theta} \approx \frac{1}{\sqrt{p}}\left(1+\frac{p}{2} Q^{2} \sin ^{2} \theta\right),
$$

and so we see that, compared to the neutral black hole case, the vortex is thicker on the equatorial region when the black hole has charge. This effect is of order $p \sim 1 / N$ and acts against the "squeezing" of the string due to the black hole attraction. Intuitively, the presence of charge induces tension-a repulsive effect.

However, there is a more important modification introduced by a nonzero charge on the black hole. If we compute the magnetic flux crossing any portion of the horizon, which, from Eq. (2.19), is given by

$$
\left.F_{\theta \varphi}\right|_{r=r_{+}}=-p\left(r_{+}^{2}-Q^{2}\right) \sin 2 \theta
$$

we see that it decreases as we increase the charge, until it precisely vanishes for an extreme black hole. Moreover, we see from Eq. (2.23) that the Higgs field also vanishes at the horizon in that limit. The extreme black hole expels from its horizon all the fields that reside in the core of the string.

It was already known that an extreme black hole placed in a uniform magnetic field exhibits a sort of "perfect diamagnetism." The solution (2.19) for the gauge field describes precisely this effect. But here we have found that this exclusion is also true for the Higgs field associated with the string vortex. Moreover-and this is something that we could not have anticipated from what we knew about the behavior of the magnetic fields - a global string is also expelled from the extreme horizon. This is very easy to see: simply set $p$ $=0$ in Eq. (2.23) to obtain the field of the global string.

Given that the solution we have found is only a leadingorder approximation for large $N$, one might inquire whether further corrections still preserve the expulsion of the fields. The numerical evidence from next section confirms this point, even down to $N=1$.

A natural question to ask is whether the black hole will stay inside the vortex or will instead try to find its way outside the core. To this effect we will study the energy stored in the string core when a black hole is sitting inside it.

For a static solution of the Abelian Higgs equations, in length and energy units rescaled by the Higgs wavelength, the energy density takes the form 


$$
T_{0}^{0}=-g^{i j} \partial_{i} X \partial_{j} X-X^{2} g^{i j} P_{i} P_{j}+\frac{\beta}{2} F^{2}+\frac{1}{4}\left(X^{2}-1\right)^{2} .
$$

More specifically, for the vortex in the Reissner-Nordström background,

$$
\begin{aligned}
T_{0}^{0}= & V\left(\partial_{r} X\right)^{2}+\frac{1}{r^{2}}\left(\partial_{\theta} X\right)^{2}+\frac{N^{2} X^{2} P^{2}}{r^{2} \sin ^{2} \theta}+\frac{1}{4}\left(X^{2}-1\right)^{2} \\
& +\frac{N^{2} \beta}{r^{2} \sin ^{2} \theta}\left[V\left(\partial_{r} P\right)^{2}+\frac{1}{r^{2}}\left(\partial_{\theta} P\right)^{2}\right] .
\end{aligned}
$$

Let $\mathcal{E}_{0 \text {,bh }}^{(g)}$ be the total energy of the gauge field in the absence or presence of the black hole. A rather long analysis, which we will spare the reader, keeping leading-order terms in $1 / N$ (or, what amounts to the same, expanding for small $p$ ) leads to the conclusion that

$$
\frac{\mathcal{E}_{\mathrm{bh}}^{(g)}}{\mathcal{E}_{0}^{(g)}}=1-c E \sqrt{p}+O(p)
$$

where $c$ is a positive constant (a pure number) of order unity. Hence the presence of a black hole within the vortex decreases the energy of the gauge field. For fixed black hole mass $E$, this is independent of the value of the charge. Even if the latter causes an equatorial thickening of the string which would tend to increase the energy, the energy of the fields decreases. This is, however, a smaller effect of order $O(p)$.

Consider now the energy stored in the Higgs field. From the solution (2.23) we can see that switching on a black hole mass $E$ decreases the value of the Higgs field inside the vortex. Again, the charge works in the opposite direction, but this is a smaller effect. As regards the energy, the largest contribution is the potential energy arising from the fact that the core is in the false vacuum. This is, however, hardly affected by the introduction of the black hole. The gradient terms, on the other hand, are more significantly modified, and it is not difficult to see that a nonzero value of the black hole mass $E$ always tends to decrease the energy.

Of course, these energetic considerations alone do not tell us what the forces induced by the vortex on the black hole are. In order to compute these, we would need to consider configurations where the black hole is not exactly at the axis of the vortex and, thus, nonaxisymmetric configurations. A simple way to estimate the forces would be to compute the energy stored in the vortex as a function of the separation $x$ of the center of the black hole to the axis of the vortex; call this function $\mathcal{E}(x)$. It is clear that the lack of symmetry makes this problem very much harder. Nonetheless, the estimations above give us the values $\mathcal{E}(x=0)=\mathcal{E}_{\mathrm{bh}}<\mathcal{E}(x$ $\rightarrow \infty)=\mathcal{E}_{0}$. If $\mathcal{E}(x)$ were a monotonic function of $x$, which does not seem unreasonable, then the forces acting on the black hole would tend to keep it inside the vortex.
The conclusion seems to be that the black hole should remain stable inside a thick vortex. Qualitatively, this is largely independent of the presence or absence of black hole charge and, in particular, of the vanishing of the fields on an extreme horizon. However, as will be revealed in next section, this no longer remains true if the vortex radius shrinks below the horizon radius.

\section{NUMERICAL SOLUTIONS}

In the previous section we have provided evidence that an extremal black hole does not allow penetration through its horizon of the fields associated with the vortex. The analysis, though, has had to be restricted to the situation where the black hole is small relative to the vortex and stays well within its core. It is irresistible to push this picture to its limits and let the vortex shrink to a size smaller than the horizon radius. Will the string still fail to pierce the extreme horizon? In this case we would expect that the presence of the black hole inside the vortex should cause an increment of the tension of the flux. As a result, the energy stored in the vortex should increase-instead of decrease, as in the previous section-and this would clearly suggest that the configuration is unstable: the extremal black hole would strongly oppose wearing the Abelian Higgs wig, and the (thin) string should slide off the horizon, leaving the extreme black hole as bald as we have always known it to be.

To analyze these issues we shall need to resort to numerical integration of Eqs. (2.13) and (2.14) outside and on the black hole horizon. Our results will confirm the picture of the previous section for thick vortices, as well as provide evidence that, when the string is thin, it will tend to slip off the extreme horizon.

The Abelian Higgs equations in the presence of a background Reissner-Nordström metric are elliptic. On the horizon they become parabolic. In order to solve the equations numerically, we use a technique first used by Achúcarro, Gregory, and Kuijken [8]. We will briefly describe this technique below.

One common approach to solving elliptic equations is to introduce an artificial, first-order in time, diffusive term to the elliptic equation to be solved. The resulting diffusion equation is then iterated and the fields relaxed, until the timedependent term (the "residual") approaches zero to sufficient accuracy, leaving a solution to the original elliptic equation. This is the basic technique used in [8]; however, they have introduced some changes in order to solve the equations on the horizon.

Their method is to set boundary conditions at $\theta=0$ and $\theta=\pi$ consistent with field values at an Abelian string core. At $r=\infty$, boundary conditions are set to those of the asymptotic fields of the string. Field values on the horizon are also initially set to asymptotic values. The integration technique then proceeds as follows.

First, the discretized field is relaxed inside the simulation volume. Next, using the equations for the fields on the horizon, which are elliptic in the radial direction, the field is relaxed on the horizon, giving new boundary points there. This process is iterated until the residual is considered small 
enough that a solution has been found.

The relaxation procedure we have used is based on the successive overrelaxation method described in [14]. However, since the equations are nonlinear, Chebyshev acceleration had to be turned off, and we typically had to underrelax the field.

To check our code, we ensured that solutions to the uncharged black hole case matched those of [8].

The discretized equations for the $P$ and $X$ fields in a Reissner-Nordström background outside the horizon are

$$
\begin{gathered}
X_{00}=\frac{\frac{2}{r}\left(1-\frac{E}{r}\right) \frac{X_{+0}-X_{-0}}{2 \Delta r}+\frac{\cot \theta}{r^{2}} \frac{X_{0+}-X_{0-}}{2 \Delta \theta}+\left(1-\frac{2 E}{r}+\frac{Q^{2}}{r^{2}}\right) \frac{X_{+0}+X_{-0}}{\Delta r^{2}}+\frac{X_{0+}+X_{0-}}{r^{2} \Delta \theta^{2}}}{\left(1-\frac{2 E}{r}+\frac{Q^{2}}{r^{2}}\right) \frac{2}{\Delta r^{2}}+\frac{2}{r^{2} \Delta \theta^{2}}+\frac{1}{2}\left(X_{00}^{2}-1\right)+\left(\frac{N P_{00}}{r \sin \theta}\right)^{2}+\frac{2}{r^{2} \Delta \theta^{2}}+\frac{1}{2}\left(X_{00}^{2}-1\right)+\left(\frac{N P_{00}}{r \sin \theta}\right)^{2}}, \\
P_{00}=\frac{\frac{2}{r^{2}}\left(E-\frac{Q^{2}}{r}\right) \frac{P_{+0}-P_{-0}}{2 \Delta r}-\cot \theta \frac{P_{0+}-P_{0-}}{2 r^{2} \Delta \theta}+\left(1-\frac{2 E}{r}+\frac{Q^{2}}{r^{2}}\right) \frac{P_{+0}+P_{-0}}{\Delta r^{2}}+\frac{P_{0+}+P_{0-}}{r^{2} \Delta \theta^{2}}}{\left(1-\frac{2 E}{r}+\frac{Q^{2}}{r^{2}}\right) \frac{2}{\Delta r^{2}}+\frac{2}{r^{2} \Delta \theta^{2}}+\frac{X_{00}^{2}}{\beta}},
\end{gathered}
$$

and the $P$ and $X$ equations on the horizon are

$$
\begin{aligned}
& X_{00}=\frac{\sqrt{E^{2}-Q^{2}} \frac{X_{+0}}{\Delta r}+\frac{X_{0+}+X_{0-}}{2 \Delta \theta^{2}}+\cot \theta \frac{X_{0+}-X_{0-}}{4 \Delta \theta}}{\frac{\sqrt{E^{2}-Q^{2}}}{\Delta r}+\frac{1}{\Delta \theta^{2}}+\frac{r_{+}^{2}}{4}\left(X_{00}^{2}-1\right)+\frac{1}{2}\left(\frac{N P_{00}}{\sin \theta}\right)^{2}}, \\
& P_{00}=\frac{\sqrt{E^{2}-Q^{2}} \frac{P_{+0}}{\Delta r}+\frac{P_{0+}+P_{0-}}{2 \Delta \theta^{2}}-\cot \theta \frac{P_{0+}-P_{0-}}{4 \Delta \theta}}{\frac{\sqrt{E^{2}-Q^{2}}}{\Delta r}+\frac{1}{\Delta \theta^{2}}+\frac{r_{+}^{2}}{4 \beta} X_{00}^{2}} .
\end{aligned}
$$

Here a zero subscript indicates the value at a given meshpoint, and + and - indicate adjacent values to the left or right.

On the $\theta=0, \pi$ boundaries, we set $P=1$ and $X=0$, at $r$ $=r_{\max }$ we set $P=0$ and $X=1$, and initially, on the horizon, we set $P=0$ and $X=1$. The boundary conditions at $r=r_{\max }$ are only an approximation to the correct values since the string is forced to have a width of one grid zone at $r_{\max }$. This tends to distort the field values near $r_{\max }$. In our simulations, we have solved the equations on a Cartesian $r-\theta$ mesh. In order to minimize the distortion, we set $r_{\max }$ to be from 5 to 10 horizon radii. Since grid zone volume increases for large $r$, the string is then well approximated as having a width of less than a grid zone in $r-\theta$ coordinates.

With the above discussion in mind, we now present the numerical results.

\section{A. Expulsion of the electromagnetic and Higgs fields by the extreme black hole}

We have already seen, in Eqs. (2.23)-(2.26), that when the vortex size is large compared to the black hole size the magnetic and Higgs fields are both expelled by the extreme black hole. However, the estimates which we used to obtain these analytic expressions no longer hold when the vortex is very thin relative to the hole. In this situation, we have to use numerical techniques.

We have pushed this calculation to the limits, making the vortex as small as we could given the computational constraints. What we have found is that the vortex is always expelled, no matter how small the magnetic and Higgs flux tubes are taken to be.

Here we present dramatic pictures of the numerical evidence which we have amassed. Our intention is to give the reader a "flavor" of the general phenomena using a frugal selection of images. The general pattern displayed here holds no matter how small you make the flux tubes.

We begin with the expulsion of the $P$ field by the extreme hole. In the diagram below, we have set $E=Q=10$, with winding number $N=1$ (the smallest winding possible). Furthermore, the Bogomol'nyi parameter $\beta$ is set equal to unity, so that the magnetic and Higgs flux tubes are the same size (see Fig. 1).

Clearly, the $P$ field literally "wraps" the black hole horizon; furthermore, given the relation between $P$ and $F_{\theta \varphi}$, it is clear that no magnetic flux is crossing the horizon. The extreme hole still behaves just like a perfect diamagnet. We now want to see if we can "puncture" the horizon with flux by making the magnetic flux tube even smaller. Perhaps the simplest way to make the vector flux tube thinner is by decreasing the value of $\beta$. This has the effect of greatly enhancing the size of the mass term in Eq. (2.14). Since $\beta$ is the ratio of the sizes of the vector and Higgs flux tubes, making $\beta$ very small will correspond to making the magnetic flux 


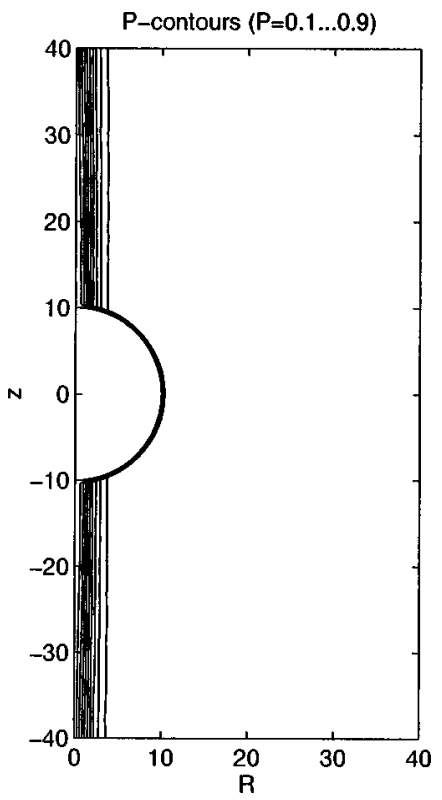

FIG. 1. Expulsion of the $P$ field from the extreme horizon, for the values $E=Q=10, N=1$, and $\beta=1$.

tube very thin (while keeping the size of the Higgs flux tube fixed, though enlarging the size of the transition region between massless and massive Higgs phases, which is $\sim \beta^{-1 / 2}$ ). This is done in Fig. 2, where we have set $E=Q$ $=10, N=1$, and $\beta=0.0001$.

Again, the $P$ contours all wrap around the black hole horizon, indicating that there is never any penetration. We have repeated this calculation for the smallest resolvable values of $\beta$ (keeping $E, Q$, and $N$ fixed), and we have always seen the same phenomena. Similarly, we have kept $N$ and $\beta$ fixed and made $E=Q$ very large (i.e., fixed the vortex size and in-

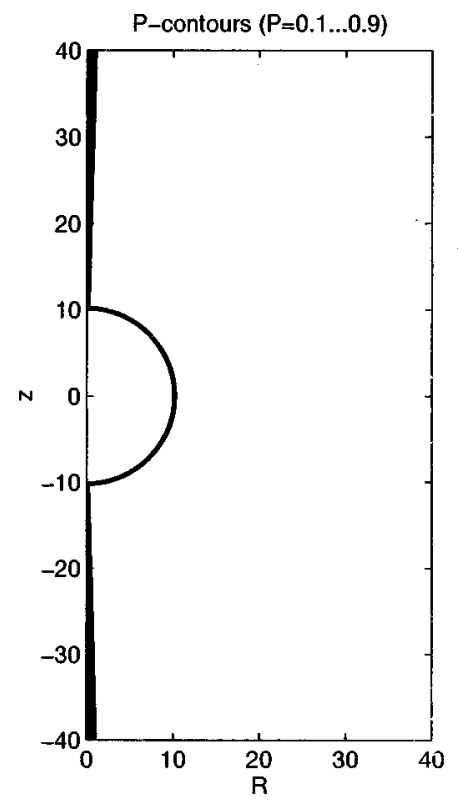

FIG. 2. Expulsion of the $P$ field from the extreme horizon, for the values $E=Q=10, N=1$, and $\beta=0.0001$. creased the size of the extreme hole), and again we see perfect expulsion. (There is very little reason to show the pictures of these calculations since without magnification they are qualitatively identical to the figure above.)

We now turn to the behavior of the Higgs field $X$. Again, in the thin vortex limit we are unable to make analytic estimates and we are forced to resort to numerical integration. We have found that the $X$ field is always expelled from the extreme hole, no matter how small the scalar flux tube is made. Actually, in Fig. 3 what we do is fix the size of the scalar flux tube (by fixing $N=1$ and $\beta=0.5$ ) and we allow the mass of the extreme hole to increase. The plots run from left to right with increasing mass. The graphs are plotted for the values $E=Q=1, E=Q=5, E=Q=10$, and $E=Q=20$.

As was claimed, the $X$ contours all wrap around the black hole horizon, no matter how large the hole is made. Indeed, the sequence of pictures in Fig. 3 provides an intuitive picture of why Eq. (2.29) makes sense. When the black hole is much smaller than the vortex, the black hole is just a "hole" where no vortex energy can be stored. Thus the presence of the hole tends to subtract the total energy of the vortex. On the other hand, when the hole becomes much larger than the vortex (and our estimates break down), the vortex still has to wrap the hole and so we would expect the total energy of the vortex to become very large. We now provide a more detailed discussion which will show that this intuition is in fact correct.

\section{B. Instability of the vortex energy in the large mass limit}

As we have discussed, Eq. (2.29) tells us that when the hole is small relative to the vortex, increasing the mass of the hole tends to decrease the total energy stored in the vortex. We can also see it must be the case that when the hole is very large relative to the vortex, increasing the mass of the hole must increase the energy of the vortex due to the tension in the flux lines. Thus the energy of the vortex as a function of extreme black hole mass must have at least one minimum. In fact, it is not hard to see that there must be at most one minimum (although we will not provide an analytic argument here, since the numerical results will make this clear). We shall denote this value of the hole mass, where the vortex energy is minimized, as $E_{c}(N, \beta)$. We have written $E_{c}$ as a function of $N$ and $\beta$ in order to emphasize that the critical mass depends on the "width" of the vortex. Now, again, let $\mathcal{E}_{\mathrm{bh}}$ denote the total energy of the vortex centered on the extreme black hole (note that in the numerical calculations which follow we have introduced an obvious cutoff; i.e., we do not integrate over all of spacetime to obtain the energy, but rather we integrate out to the boundaries of some large "box"). Then it is always the case that $\mathcal{E}_{\mathrm{bh}}\left(E_{c}\right)<\mathcal{E}_{0}$, where $\mathcal{E}_{0}$ is the energy of the vortex in the absence of the black hole. This means that a black hole of mass $E_{c}$ is perfectly happy to sit inside of the vortex, and indeed it would be energetically unfavorable for the hole to be removed from the vortex. In fact, it is always the case that there exists a maximum mass $E_{\max }$ such that for all black holes of mass $E<E_{\max }, \mathcal{E}_{\mathrm{bh}}(E)<\mathcal{E}_{0}$; as long as the hole is not too massive, it is content to sit inside the vortex. 

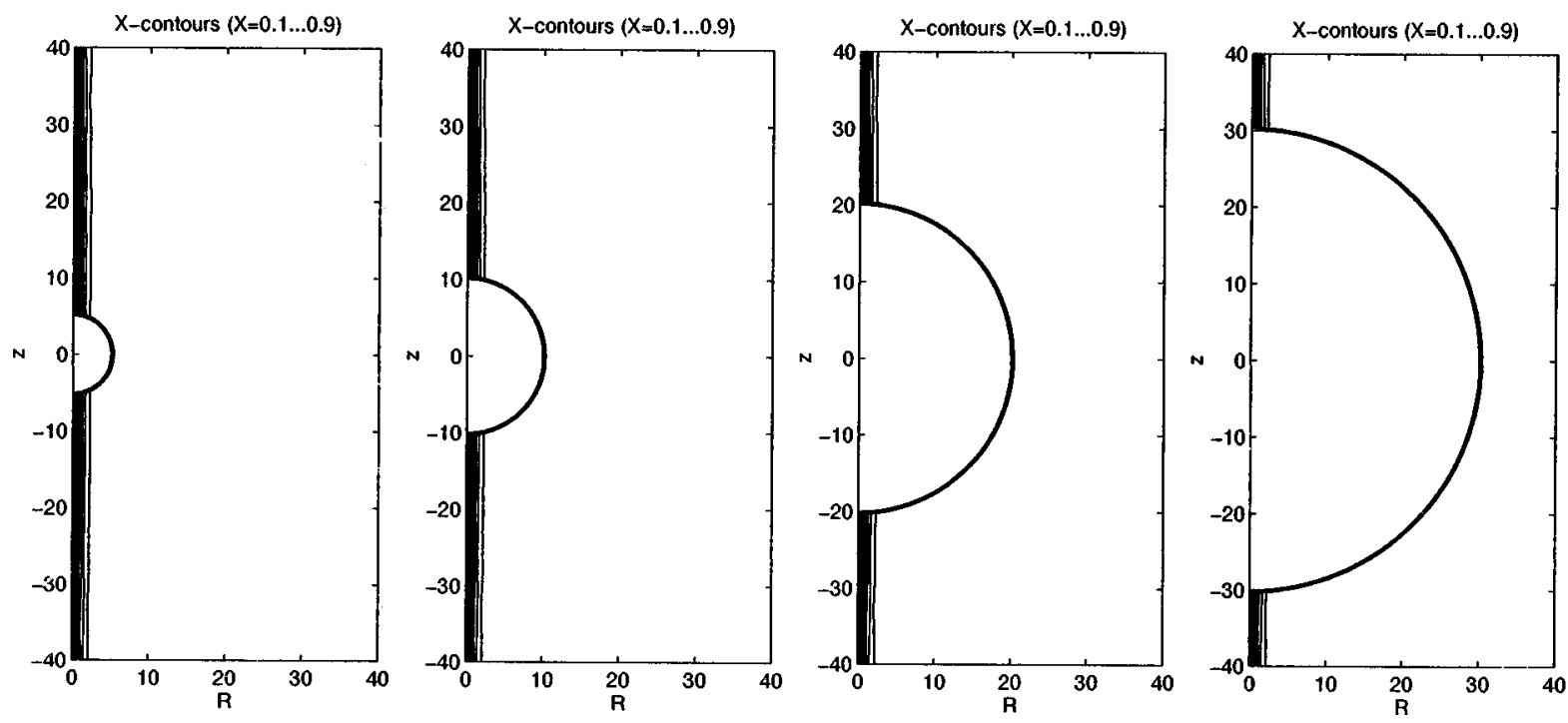

FIG. 3. Expulsion of the Higgs field from the extreme horizon, for the values (from left to right) $E=Q=5,10,20,30, N=1$, and $\beta=0.5$.

The statements made above are based on the results of our numerical computations of the total energy $\mathcal{E}_{\mathrm{bh}}$. In the figure below we have plotted the results of one such computation. Here we have set $\beta=0.5$ and $N=10$. The flat, horizontal line (at 6640) represents $\mathcal{E}_{0}$ in our units. Clearly, for these values $E_{c}$ is about 8 and $E_{\max }$ is about 15. Furthermore, for black holes of mass greater than $E_{\max }$, the energy of the vortex is diverging. The erratic behavior of the vortex energy for very small values of the black hole mass is an artifact of the numerical techniques employed in the calculation and should be ignored (see Fig. 4).

It is clear from the graph of Fig. 4 that a black hole with mass $E>15$ is going to find it energetically favorable to slip out of the vortex. Thus it is really not appropriate to think of such a vortex as a "property of the black hole"; the identification of the vortex as long hair does not seem to go

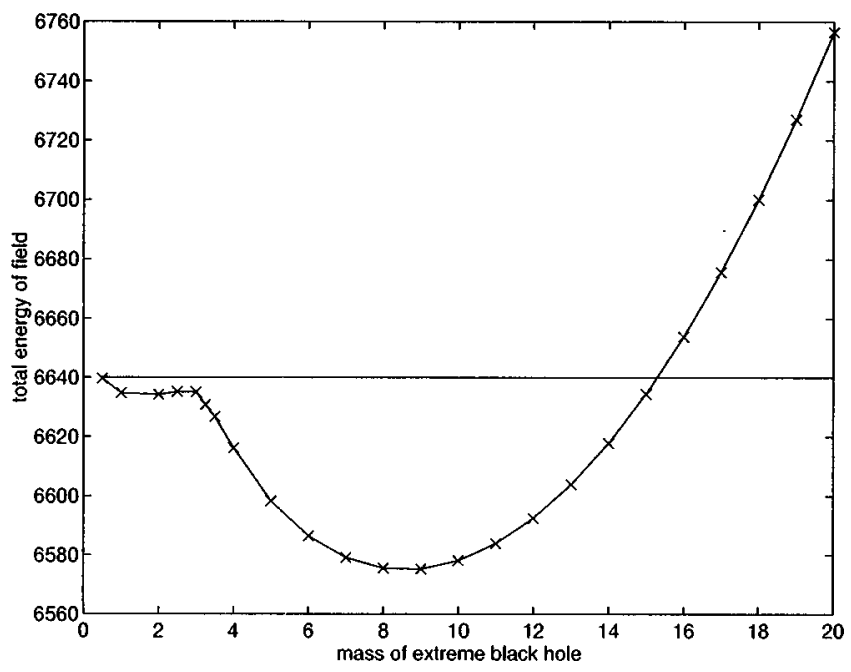

FIG. 4. Plot of total vortex field energy as a function of black hole mass. through in this situation. Of course, when the mass of the hole is small, you could still technically try to identify the vortex with hair since at least in that case the configuration is energetically stable. On the other hand, the fact remains that the vortex is completely expelled from the hole, even in the (putatively) stable situation. Thus one would say that the vortex is not dressing the black hole. It is still unclear to us whether or not one should think of such a "thick" vortex as genuine hair for a small extreme black hole. This is somewhat a reversal of previously studied situations (e.g., the colored black holes), where the black hole may be dressed, but the configuration is unstable.

\section{No expulsion of vortex fields in the near-extreme limit}

So far, we have presented firm numerical evidence that the fields of an Abelian Higgs vortex are expelled from the horizon of an extreme black hole. A natural question is then whether or not similar results continue to hold when the hole is made slightly nonextreme. As is well known, a nonextreme black hole with nonsingular horizon has $Q<E$. As we let $Q$ approach $E$ from below (letting the hole approach extremality), will we see the fields $P$ and $X$ "gradually" expelled from the horizon? Or will the fields suddenly "pop" out only when we get precisely to the extreme limit?

In order to understand how to answer this question, it is useful to first recall the estimates which we made in Sec. II in the limit where the vortex is thick compared to the outer horizon radius of the black hole. In particular, recall Eq. (2.26), which follows immediately from Eq. (2.19). Equation (2.26) tells us that, in regions where the mass of the gauge field is negligible, the magnetic flux across the horizon in the nonextreme limit will always be nonvanishing and, hence, that the vortex $P$ field will penetrate the horizon. The flux vanishes in the extreme limit since the equation says that $F_{\theta \varphi}$ on the horizon is proportional to $r_{+}^{2}-Q^{2}$ (where $r_{+}$is the 


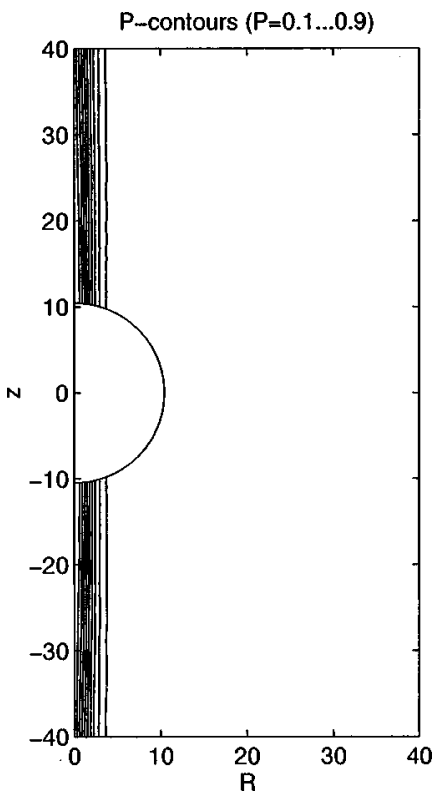

FIG. 5. Penetration of a non-extremal horizon by the $P$ field, for the values $E=10, Q=9.99, N=1$, and $\beta=1$.

outer horizon radius) and $r_{+}=Q$ precisely in the extreme limit.

Now the region $r_{-}<r<r_{+}$is regular in the coordinates which we have been using, even though there are (removable) coordinate singularities at the inner and outer horizons $r_{-}$and $r_{+}$. Furthermore, there is no reason why the estimate (2.26) should not continue to hold in this region. In other words, there is always a surface at $r=Q$, which we dub the "Meissner surface," across which no flux may flow. This Meissner surface agrees with the outer black hole horizon only in the extreme limit, and so it is only in this limit that the Meissner surface is of relevance to external observers. One could think that, since for a near-extremal black hole the Meissner surface can be very close to the (outer) horizon, then if the layer of vortex on the Meissner surface is thick enough, the expulsion from the Meissner surface might be appreciable by external observers. Now this vortex layer gets thicker with vortex size. But for large vortices, the effect of the Meissner surface can be read from Eq. (2.19), and we see that the expulsion only appears when the extremal limit is reached.

In all of our numerical calculations, we do not consider the penetralia of the black hole. Rather, we solve for test fields outside (and on) the horizon of the hole and we do not concern ourselves with what goes on inside the horizon. This is why, by construction, we do not expect to see the fields gradually expelled from the horizon.

For the edification of the reader we present here some pictures of calculations which show that the argument given above goes through even when the vortex is thin relative to the radius of the hole. In Fig. 5, where we plot $P$, we have set $E=10, Q=9.99, N=1$, and $\beta=1$.

Clearly, the $P$ field is passing right through the black hole horizon even though the hole is quite close to extremality. Similarly, one finds that the $X$ field contours flow through the

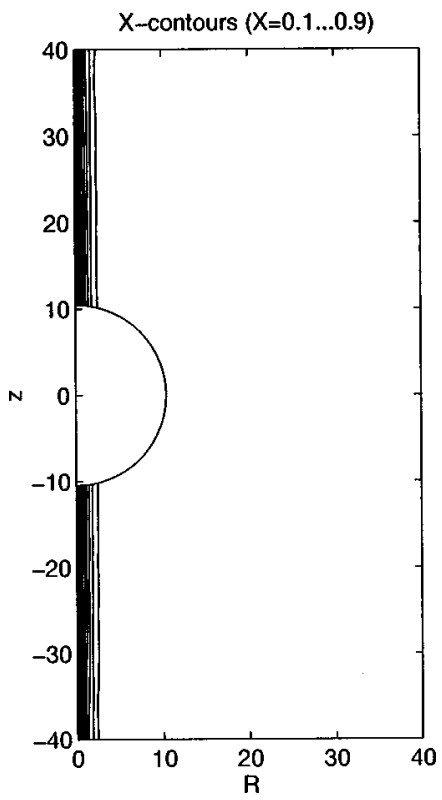

FIG. 6. Penetration of a non-extremal horizon by the Higgs field, for the values $E=10, Q=9.99, N=1$, and $\beta=1$.

outer horizon of any nonextreme black hole. In Fig. 6, where we plot $X$, we have again set $E=10, Q=9.99, N=1$, and $\beta=1$.

\section{CONCLUSIONS}

We have found that the fields of a vortex are always expelled from an extreme horizon; this effect is generic for arbitrary relative sizes of the horizon and the vortex core. Furthermore, a thin enough vortex tends to slip off the black hole. Thus it appears that an extreme black hole cannot support "long" Abelian Higgs hair. Of course, we have in no way accounted for the back reaction of the vortex on the geometry. Is there any reason why the flux tubes should not pierce the horizon once the back reaction is included? Actually, there is a piece of evidence that the expulsion may hold exactly: there do exist exact solutions (i.e., including the full back reaction) for black holes in $\mathrm{U}(1)^{2}$ theories where a black hole that is charged to extremality with respect to one of the gauge fields completely expels the field of a (Melvin) flux tube of the other gauge field [11]. In these solutions none of the gauge symmetries is broken, but recall that the spontaneous symmetry breaking is of negligible influence on the perturbative first-order solution inside the core that we have found in Eq. (2.19). This strongly suggests that, after accounting for the back reaction, the flux should be expelled from a black hole that sits inside it, at least in the case where the vortex is thick. In view of the evidence provided above, the effect could as well persist for thinner black holes, but we cannot be conclusive. In any case, the back reaction would certainly be expected to be small if the energy scale of symmetry breaking is sufficiently small compared to the black hole mass.

In order to implement a back reaction in the numerical calculations, we would first start with a fixed background and solve for the "test fields" as we have done in this paper. 
Next, we would have to plug the energy-momentum tensor for the test fields into the Einstein equations and solve for the "corrected" background geometry. Then we would again have to solve for the vortex field configuration in the corrected geometry, and so on. Now, in general, the horizon will move each time we obtain a corrected background geometry. While we are currently working on a numerical approach to include the back reaction, we will have nothing more to say about this issue here.

If, as we have argued, the vortices may fail to penetrate extreme horizons, then there are several interesting implications. Consider what happens when a string tries to end at a black hole. It has been argued in $[8,17]$ that there is no global topological obstruction for a topologically stable string to end at a black hole. The reason is that the spatial topology $S^{2} \times \mathbf{R}$ of the extended black hole spacetime allows one to take gauge patches in the manner of $\mathrm{Wu}$ and Yang that transform the trivial vacuum on one side of the black hole to the nontrivial configuration of the vortex on the other side. Furthermore, there do exist solutions where the string actually penetrates the nonextreme black hole, as shown for Schwarzschild spacetime in [8] and generalized to a Reissner-Nordström background in this paper. Now consider what happens if the black hole is extremally charged with respect to a different $\mathrm{U}(1)$. Then, again, there is no topological obstruction in principle, since the topology of the spatial sections is still $S^{2} \times \mathbf{R}$ and this would admit a gauge patching of the same sort as before. But what we have found seems to strongly suggest that, even if the penetration on only one side of the black hole is globally topologically feasible, there does not exist a solution of the equations of motion that does actually penetrate. We say "suggest" since we have not analyzed the situation where the string is only on one side of the black hole, but our results very strongly hint that there is no way a vortex can penetrate an extreme horizon: the reason, we would say, is that this penetration is a local issue, not having to do with global topological considerations. Now, if the string cannot pierce the extreme horizon, then there is no way that one can construct the Wu-Yang type of patch for the string to end at the black hole: intuitively, there is no place for the flux to escape. With the caveats above in mind-back reaction being perhaps the most troublesome issue-it would follow that a topologically stable string cannot terminate on the horizon of a black hole that is extremally charged relative to a distinct, unbroken $\mathrm{U}(1)$. This is a rather unexpected twist, since one usually assumes, naively, that once the topological obstruction disappears, the desired solution can be constructed.

Now there have been a number of papers describing the pair creation of black holes with strings ending on them [1519]. Apart from the topological stability issues, the process of a string snapping with formation of black holes differs in one important respect from the strings that break with monopoles at the end. In order for the Euclidean gravitational instanton that mediates the process to be regular, the black holes must have (unconfined) charge and be either extremal or close to extremality. This forces one to introduce, in addition to the massive gauge field carried by the string, a (massless) U(1) field to which the black hole charge couples.
Effectively, one works in a $\mathrm{U}(1)^{2}$ theory of the same kind we have been discussing in this paper. But if, as we have argued, the string cannot end at the extreme horizon, the corresponding instanton does not exist. This would seem to imply that a Nielsen-Olesen string could not snap by forming extreme black holes at its ends. Therefore, consideration of "realistic" strings seems to impose new selection rules on string snapping, of a sort somewhat different from those recently discussed in [20].

Furthermore, if the extrapolation of our no-penetration results to strings trying to end at an extreme black hole were correct, then another thing that the string could not do is to "fray" - as discussed in [17] — by forming extreme black holes on it, since in order for the string to fray the tension, and thus the flux, must be different on each side of the black hole. But the flux cannot be different on each side for reasons identical to those just discussed: without piercing the black hole, there is no place for the "excess" flux to go.

Another interesting scenario involving pair creation of black holes, still in a theory with two gauge fields $\mathcal{F}$ (massless) and $F$ (massive), is the following: let there be a string vortex (carrying confined flux of $F$ ) and a magnetic (unconfined) background field $\mathcal{B}$ parallel to the string. Suppose that a pair of magnetic holes, charged relative to the $\mathcal{B}$ field with charges $\pm q$, are pair created and accelerate apart under the force induced by the field, like in the Schwinger pair creation process. Suppose, moreover, that the black holes are created right on the string, but that the latter does not snap or "fray." This process can be described by means of the Ernst metric with a constant conical deficit along the axis where the black holes lie.

In principle, the presence of the string does affect the pair creation rate: it is enhanced relative to the creation of black holes away from the string, since the action of the instanton is smaller precisely by a factor of the conical deficit. This enhancement is no more than the effect (discussed in [19] in the context of thermal nucleation of black holes) that a black hole nucleates preferentially on a string, rather than on flat space. Now, if the holes are extreme, the string cannot penetrate the horizon of either of the holes. Rather, the vortex must wrap around each of the black hole horizons, so that the entire configuration will look rather like two peas in a pod being squeezed apart. Now suppose that the created holes are much larger than the vortex flux tube. Then the created holes will want to pop out of the vortex. This would suggest that the rate at which two extreme black holes nucleate on a (nonsnapping) string will be strongly suppressed and probably zero. It would also suggest that a one-dimensional interacting gas of small extreme black holes would populate the string. Research on this and related problems is currently underway.

Note added in proof. After this work was completed, and accepted for publication, more recent studies have appeared [21], which cast doubt on our numerical results for thin vortices in the extreme black hole background. As a result, thin enough vortices seem to be capable of piercing extremal horizons. However, the expulsion seems to be a true phenomenon for thicker vortices. In particular, we believe the analytical results in Sec. II to be valid. 


\section{ACKNOWLEDGMENTS}

The authors would like to thank Ana Achúcarro, Fay Dowker, and Simon Ross for useful conversations and Ruth Gregory for carefully reading the manuscript. A.C. was supported by Grant No. NSF PHY94-07194 at ITP, Santa Bar- bara, and by Pembroke College, Cambridge. J.M.A.A.-C. was supported by Wolfson College, University of Oxford. R.E. was partially supported by FPI (MEC-Spain) and by Grant No. UPV 063.310-EB225/95. A.S. was supported by UK PPARC Grant No. GR/L21488.
[1] W. Israel, Phys. Rev. 164, 1776 (1967).

[2] R. B. Wald, Phys. Rev. Lett. 26, 1653 (1971).

[3] B. Carter, Phys. Rev. Lett. 26, 331 (1971).

[4] R. Bartnik and J. McKinnon, Phys. Rev. Lett. 61, 141 (1988).

[5] P. Bizon and R. M. Wald, Phys. Lett. B 267, 173 (1991).

[6] R. Metsaev and A. Tseytlin, Nucl. Phys. B293, 385 (1987).

[7] P. Kanti, N. E. Mavromatos, J. Rizos, K. Tamvakis, and E. Winstanley, Phys. Rev. D 54, 5049 (1996).

[8] A. Achúcarro, R. Gregory, and K. Kuijken, Phys. Rev. D 52, 5729 (1995).

[9] A. Vilenkin and E. P. S. Shellard, Cosmic Strings and Other Topological Defects (Cambridge University Press, Cambridge, England, 1994); M. B. Hindmarsh and T. W. B. Kibble, Rep. Prog. Phys. 58, 477 (1995).

[10] M. Aryal, L. Ford, and A. Vilenkin, Phys. Rev. D 34, 2263 (1986).

[11] A. Chamblin, R. Emparan, and G. W. Gibbons, "On the Superconducting Properties of $p$-Branes and Extremal Black
Holes," DAMTP report (in preparation).

[12] H. B. Nielsen and P. Olesen, Nucl. Phys. B61, 45 (1973).

[13] A. Achúcarro, R. Gregory, J. Harvey, and K. Kuijken, Phys. Rev. Lett. 72, 3646 (1994).

[14] W. H. Press, S. A. Teukolsky, W. T. Vetterling, and Brian P. Flannery, Numerical Recipes in Fortran (Cambridge University Press, Cambridge, England, 1992).

[15] S. W. Hawking and S. R. Ross, Phys. Rev. Lett. 75, 3382 (1995).

[16] R. Emparan, Phys. Rev. Lett. 75, 3386 (1995).

[17] D. M. Eardley, G. T. Horowitz, D. A. Kastor, and J. Traschen, Phys. Rev. Lett. 75, 3390 (1995).

[18] R. Gregory and M. Hindmarsh, Phys. Rev. D 52, 5598 (1995).

[19] R. Emparan, Phys. Rev. D 52, 6976 (1995).

[20] A. Achúcarro and R. Gregory, Phys. Rev. Lett. 79, 1972 (1997).

[21] F. Bonjour and R. Gregory, hep-th/9809029. 